\title{
LEVEL STRIPPING FOR DEGREE 2 SIEGEL MODULAR FORMS
}

\author{
RODNEY KEATON
}

\begin{abstract}
In this paper, we consider stripping primes from the level of degree 2 cuspidal Siegel eigenforms. Specifically, given an eigenform of level $N \ell^{r}$ under certain restrictions, where $\ell \nmid N$ is a prime, we construct an eigenform of level $N$, which is congruent in eigenvalues to our original form. To obtain our results, we use constructions of Eisenstein series and theta functions to adapt ideas from a level stripping result on elliptic modular forms.
\end{abstract}

\section{Introduction}

Throughout we fix a prime $\ell \geq 5$, and let $N$ be a positive integer coprime to $\ell$. Let $K$ be a number field with $\nu$ a prime lying over $\ell$ and let

$$
\rho: \operatorname{Gal}(\overline{\mathbb{Q}} / \mathbb{Q}) \rightarrow \mathrm{GL}_{2}\left(\mathcal{O}_{K_{\nu}}\right)
$$

be a Galois representation, where $K_{\nu}$ is the completion of $K$ at $\nu$. Let $\bar{\rho}$ denote the residual representation of $\rho$ obtained by composition with the map $\mathrm{GL}_{2}\left(\mathcal{O}_{K_{\nu}}\right) \rightarrow$ $\mathrm{GL}_{2}\left(\mathbb{F}_{\nu}\right)$, where $\mathbb{F}_{\nu}$ is the residue field of $\mathcal{O}_{K}$ at $\nu$. By Serre's conjecture (Theorem $1.2,[14])$ we know precisely the conditions necessary for the semisimplification of $\bar{\rho}$ to arise from a cuspidal elliptic Hecke eigenform in the sense of Deligne [8]. Furthermore, Serre's refined conjecture $(3.2 .4$ ?, $[21])$ tells us the precise character, level, and weight of such an eigenform. Note, the equivalence of Serre's conjecture and Serre's refined conjecture is known by the work of Coleman-Voloch [7], Gross [11], Ribet [19], and others (see [10]). In the process of proving this equivalence, Ribet presented the following result, which we will be interested in extending.

Theorem 1. [20, Theorem 2.1]

Let $\ell \geq 3$ be a prime. Suppose that $f$ is an eigenform of level $\Gamma_{1}\left(N \ell^{r}\right)$ with $r>0$ and $(N, \ell)=1$. Then, there exists an eigenform of level $\Gamma_{1}(N)$ whose eigenvalues are congruent to the eigenvalues $f$ modulo $\nu$ away from the level of $f$, where $\nu$ lies over $\ell$ in some number field.

In order to transfer to the setting we will primarily be interested in, we let

$$
\rho: \operatorname{Gal}(\overline{\mathbb{Q}} / \mathbb{Q}) \rightarrow \operatorname{GSp}_{4}\left(\mathcal{O}_{K_{\nu}}\right)
$$

be a Galois representation, where we are keeping the same notation as above. In this setting, there is a conjecture of Herzig and Tilouine which gives conditions on when the residual representation should arise from a degree 2 Siegel eigenform; see Section 7 for details. Given a conjecture of this form it is natural to want some type of refined

Received by the editors July 16, 2012.

2000 Mathematics Subject Classification. Primary 11F33, 11F46, Secondary 11F80.

Key words and phrases. Congruence of modular forms, Galois representations, Siegel modular forms. 
conjecture to make precise the character, level, and weight of such an eigenform. The desired weight is discussed in detail in [13]. Concerning the level, a natural starting place is Theorem 8, which is our analogue to Theorem 1 in the degree 2 setting.

Similar results have been obtained using an extension of Hida theory to Siegel modular forms; see Theorem 3.2 in [23]. However, the proof of Theorem 1 given by Ribet uses strictly classical methods. It is this approach that we adapt to the degree 2 setting. Note, we also do not require an ordinarity assumption for our argument, which is necessary for the proof of Theorem 3.2 in [23].

\section{Preliminaries}

In this section, we will introduce some basic facts about Siegel modular forms. For more details the interested reader is referred to [2].

Let $n>1$ be an integer. Let $\mathfrak{h}_{n}$ denote the Siegel upper half-space of degree $n$, and let $\operatorname{GSp}_{2 n}^{+}(\mathbb{Z})$ denote the set of $2 n \times 2 n$ symplectic matrices with integral entries and positive similitude factor. We have an action of $\operatorname{GSp}_{2 n}^{+}(\mathbb{Z})$ on $\mathfrak{h}_{n}$ given by,

$$
\gamma \cdot Z=(A Z+B)(C Z+D)^{-1},
$$

for $Z \in \mathfrak{h}_{n}, \gamma=\left(\begin{array}{ll}A & B \\ C & D\end{array}\right) \in \operatorname{GSp}_{2 n}^{+}(\mathbb{Z})$. Let $k$ be an integer, and $F$ be a holomorphic complex valued function on $\mathfrak{h}_{n}$. We define the weight $k$ slash operator by,

$$
\left(\left.F\right|_{k} \gamma\right)(Z):=\mu(\gamma)^{n k-\frac{n(n+1)}{2}} \operatorname{det}(C Z+D)^{-k} F(\gamma \cdot Z),
$$

where $\gamma$ is as above with similitude factor $\mu(\gamma)$ and $k$ is an integer greater than or equal to $n$. Note, we will drop the $k$ when the weight is clear.

In general, for a subgroup $\Gamma \leq \operatorname{Sp}_{2 n}(\mathbb{Z})$ of finite index, we say that $F$ is a Siegel modular form of weight $k$ and level $\Gamma$ if $(F \mid \gamma)(Z)=F$ for every $\gamma \in \Gamma$. However, we will be primarily interested in a certain subset of such functions.

Let $M \in \mathbb{Z}$ be strictly positive, and let $\chi$ be a Dirichlet character modulo $M$. We define $\mathbb{Q}(\chi)$ to be the number field obtained by adjoining all the values of $\chi$ to $\mathbb{Q}$. Define the following two "level $M$ " subgroups of $\operatorname{Sp}_{2 n}(\mathbb{Z})$,

$$
\begin{aligned}
& \Gamma_{0}^{n}(M):=\left\{\left(\begin{array}{ll}
A & B \\
C & D
\end{array}\right) \in \operatorname{Sp}_{2 n}(\mathbb{Z}): C \equiv 0 \quad(\bmod M)\right\}, \\
& \Gamma_{1}^{n}(M):=\left\{\left(\begin{array}{ll}
A & B \\
C & D
\end{array}\right) \in \Gamma_{0}(M): \operatorname{det} D \equiv 1 \quad(\bmod M)\right\} .
\end{aligned}
$$

We say that our $F$ from above is a Siegel modular form of character $\chi$, degree $n$, level $M$, and weight $k$ if it satisfies,

$$
(F \mid \gamma)(Z)=\chi(\operatorname{det}(D)) F(Z), \text { for all } \gamma \in \Gamma_{0}^{n}(M) .
$$

We denote the space of such functions by $M_{k}^{n}(M, \chi)$. From the above transformation we see that any $F \in M_{k}^{n}(M, \chi)$ is periodic with respect to symmetric $n \times n$ integral matrices. Hence, $F$ has a Fourier expansion of the form,

$$
F(Z)=\sum_{0 \leq T \in \Lambda_{n}} a_{F}(T) q^{\operatorname{tr}(T Z)}
$$

where $q:=\exp (2 \pi \sqrt{-1}), \operatorname{tr}(\cdot)$ denotes the trace, and our summation is over all halfintegral positive semi-definite matrices. Furthermore, it can be shown that $F \mid \gamma$ has 
a Fourier expansion of the above form for every $\gamma \in \operatorname{Sp}_{2 n}(\mathbb{Z})$. We say that $F$ is a cusp form if for every $\gamma \in \operatorname{Sp}_{2 n}(\mathbb{Z})$, we have $a_{F \mid \gamma}(T)=0$ whenever $T$ is not positive definite. We denote this subspace by $S_{k}^{n}(M, \chi)$. Furthermore, for a ring $R \subseteq \mathbb{C}$, we will use $M_{k}^{n}(M, \chi ; R)$ (resp. $\left.S_{k}^{n}(M, \chi ; R)\right)$ to denote the space of modular forms (resp. cusp forms) which have Fourier coefficients in $R$.

\section{Hecke operators}

It is well known that $M_{k}^{n}(M, \chi)$ admits a commutative set of linear operators known as the Hecke operators. The space of all Hecke operators is generated by the elements

$$
\begin{aligned}
T(p) & :=\Gamma_{1}(M) \operatorname{diag}\left(1_{n}, p 1_{n}\right) \Gamma_{1}(M), \\
T_{i}\left(p^{2}\right) & :=\Gamma_{1}(M) \operatorname{diag}\left(1_{i}, p 1_{n-i}, p^{2} 1_{i}, p 1_{n-i}\right) \Gamma_{1}(M),
\end{aligned}
$$

for $1 \leq i \leq n$, and $p$ ranges over all primes. We will denote the algebra generated by these operators over a ring $R$ by $\mathcal{H}_{R}$. Note, we have suppressed the $n$ from the notation as the degree should be clear from context. Furthermore, if $\Sigma$ is a finite set of rational primes, then we define $\mathcal{H}_{R}^{\Sigma}$ to be the subalgebra of $\mathcal{H}_{R}$ which is generated by the operators above with satisfy $p \notin \Sigma$.

Note that the operator $T_{n}\left(p^{2}\right)$ is sometimes referred to as the "diamond" operator. We have defined our modular forms so that every character $\chi$, degree $n$, weight $k$ form is an eigenvector for $T_{n}\left(p^{2}\right)$ with eigenvalue $p^{n(k-n-1) / 2} \chi(p)$. As a result of this, we will be primarily interested in the operators $T(p)$ and $T_{i}\left(p^{2}\right)$ for $1 \leq i<n$.

As these operators are self-adjoint with respect to the Petersson inner product, we can find an orthogonal basis of $S_{k}^{n}(M, \chi)$ which consists of simultaneous eigenvectors for all of the Hecke operators. We refer to these basis elements as eigenforms. For $F$ an eigenform, we define $\mathbb{Q}\left(\lambda_{F}\right)$ to be the extension of $\mathbb{Q}$ obtained by adjoining all of the eigenvalues of $F$. It is well known that such an extension is finite and totally real.

The following lemma provides us with a necessary result concerning the Fourier coefficients of cuspidal eigenforms.

Lemma 2. Let $F \in S_{k}^{n}(M, \chi)$ be an eigenform and define $K$ to be $\mathbb{Q}\left(\lambda_{F}, \chi\right)$. Set

$$
S_{k}^{n}(M, \chi)_{F}:=\left\{G \in S_{k}^{n}(M, \chi): \lambda_{G}(p)=\lambda_{F}(p) \text { for all } p\right\} .
$$

Then,

$$
S_{k}^{n}(M, \chi)_{F}=S_{k}^{n}\left(M, \chi ; \mathcal{O}_{K}\right)_{F} \otimes_{\mathcal{O}_{K}} \mathbb{C}
$$

Proof. By Corollary 2.1 of [23] we have

$$
S_{k}^{n}(M, \chi)=S_{k}^{n}(M, \chi ; \mathbb{Z}) \otimes_{\mathbb{Z}} \mathbb{C} .
$$

Let $\left\{F_{1}, \ldots, F_{r}\right\}$ be a $\mathbb{Z}$ basis for $S_{k}^{n}(M, \chi ; \mathbb{Z})$. By Theorem 6.1 in [12] we have for every $t \in \mathcal{H}_{\mathbb{Q}}$ that

$$
t F_{i}=\sum_{j=1}^{r} c_{i j}(t) F_{j},
$$

where $c_{i j} \in \mathbb{Q}(\chi)$. The remainder of the proof follows precisely as in the proof of Theorem 2 in $[16]$ with $\mathbb{Q}$ replaced by $\mathbb{Q}(\chi)$. 
Due to this lemma, we may suppose that the field obtained by adjoining all Fourier coefficients of an eigenform $F$ to $\mathbb{Q}$, denoted $\mathbb{Q}(F)$, is a finite extension.

When $M$ is the level of our form and $\ell \mid M$, we have that our Hecke operator $T(\ell)$ simplifies to the operator $U(\ell)$, which we define by its action on Fourier expansions,

$$
U(\ell): \sum_{0 \geq T \in \Lambda_{n}} a_{F}(T) q^{\operatorname{tr}(T Z)} \mapsto \sum_{0 \geq T \in \Lambda_{n}} a_{F}(\ell T) q^{\operatorname{tr}(T Z)} .
$$

For our main result we will need the following two properties of the $U(\ell)$ operator.

Lemma 3. [5, Theorem 1] If $\ell \| M$, the operator $U(\ell)$ is an injective map from $M_{k}^{n}(M, \chi)$ to itself.

Lemma 4. If $\ell^{2} \mid M$ and $\chi$ is defined modulo $\frac{M}{\ell}$, the operator $U(\ell) \operatorname{maps} M_{k}^{n}(M, \chi)$ to $M_{k}^{n}(M / \ell, \chi)$.

Proof. Here we have adapted a proof of Andrianov [1].

Let $F \in M_{k}^{n}(M, \chi)$. From [3] we have that the operator $U(\ell)$ is given by,

$$
F\left|U(\ell)=\ell^{\frac{n(n+1)}{2}} \sum_{S} F\right|\left(\begin{array}{ll}
1 & S \\
0 & \ell
\end{array}\right)
$$

where the summation runs over all symmetric matrices in $M_{n}(\mathbb{Z} / \ell \mathbb{Z})$. Then, we have

$$
F\left|U(\ell)=\ell^{\frac{n(n+1)}{2}} \sum_{S} F\right|\left(\begin{array}{ll}
1 & S \\
0 & \ell
\end{array}\right)=\ell^{\frac{n(n+1)}{2}} F \mid\left(\begin{array}{ll}
1 & 0 \\
0 & \ell
\end{array}\right) \sum_{S}\left(\begin{array}{ll}
1 & S \\
0 & 1
\end{array}\right) .
$$

Define the following subgroup of $\Gamma_{0}(M / \ell)$ :

$$
\Gamma(M / \ell, \ell):=\left\{\left(\begin{array}{ll}
A & B \\
C & D
\end{array}\right) \in \Gamma_{0}(M / \ell): B \equiv 0 \quad(\bmod \ell)\right\} .
$$

Then, for $\gamma \in \Gamma(M / \ell, \ell)$, it is not hard to show that

$$
F\left|\left(\begin{array}{ll}
1 & 0 \\
0 & \ell
\end{array}\right)\right| \gamma=\chi(\gamma) F \mid\left(\begin{array}{ll}
1 & 0 \\
0 & \ell
\end{array}\right) .
$$

Note, a complete set of right coset representatives for

$$
\Gamma(M / \ell, \ell) \backslash \Gamma_{0}(M / \ell)
$$

is given by

$$
\left\{\left(\begin{array}{ll}
1 & S \\
0 & 1
\end{array}\right):{ }^{T} S=S, S \in M_{n}(\mathbb{Z} / \ell \mathbb{Z})\right\} .
$$

Let $\gamma \in \Gamma_{0}(M / \ell)$, and let $S \in M_{n}(\mathbb{Z} / \ell \mathbb{Z})$ be symmetric. Set $S^{\prime}$ to be the unique symmetric matrix in $M_{n}(\mathbb{Z} / \ell \mathbb{Z})$ which is congruent to $(A+S C)^{-1}(B+S D)(\bmod \ell)$. Then, there exists $\gamma_{S} \in \Gamma(M / \ell, \ell)$ such that,

$$
\left(\begin{array}{ll}
1 & S \\
0 & 1
\end{array}\right) \gamma=\gamma_{S}\left(\begin{array}{cc}
1 & S^{\prime} \\
0 & 1
\end{array}\right)
$$


Note, such a $\gamma_{S}$ also satisfies $\chi(\gamma)=\chi\left(\gamma_{S}\right)$. Thus,

$$
\begin{aligned}
F|U(\ell)| \gamma & =\ell^{\frac{n(n+1)}{2}} \sum_{S} F \mid\left(\begin{array}{ll}
1 & 0 \\
0 & \ell
\end{array}\right)\left(\begin{array}{ll}
1 & S \\
0 & 1
\end{array}\right) \gamma \\
& =\ell^{\frac{n(n+1)}{2}} \sum_{S} F \mid\left(\begin{array}{ll}
1 & 0 \\
0 & \ell
\end{array}\right) \gamma_{S}\left(\begin{array}{cc}
1 & S^{\prime} \\
0 & 1
\end{array}\right) \\
& =\ell^{\frac{n(n+1)}{2}} \chi\left(\gamma_{S}\right) F \mid\left(\begin{array}{ll}
1 & 0 \\
0 & \ell
\end{array}\right) \sum_{S^{\prime}}\left(\begin{array}{cc}
1 & S^{\prime} \\
0 & 1
\end{array}\right) \\
& =\chi(\gamma) F \mid U(\ell) .
\end{aligned}
$$

This completes the proof.

\section{Congruences}

In this section, we will introduce two different notions of congruence between Siegel modular forms.

Let $F$ and $G$ be eigenforms of the same degree. Let $\lambda_{F}(p), \lambda_{F}\left(p^{2}, i\right)$, for $1 \leq i<n$, denote the eigenvalues of $F$ with respect to $T(p), T_{i}\left(p^{2}\right)$ respectively and similarly for $G$. Let $\Sigma$ denote a finite set of rational primes as in the previous section. Then, we say that $F \equiv_{\Sigma} G(\bmod \ell)$ if for all primes $p \notin \Sigma$ we have

$$
\begin{gathered}
\lambda_{F}(p) \equiv \lambda_{G}(p) \quad(\bmod \nu), \\
\lambda_{F}\left(p^{2}, i\right) \equiv \lambda_{G}\left(p^{2}, i\right) \quad(\bmod \nu), \text { for } 1 \leq i \leq n-1,
\end{gathered}
$$

where $\nu$ is a prime lying over $\ell$ in $\mathbb{Q}\left(\lambda_{F}, \lambda_{G}\right)$. We will refer to this congruence a congruence of eigenvalues.

Our second notion of congruence we will refer to as the congruence of Fourier coefficients, which we define as in [5]. Define the $\ell$-adic valuation of $F$ as

$$
\operatorname{ord}_{\ell}(F):=\inf _{T}\left\{\operatorname{ord}_{\ell}\left(a_{F}(T)\right)\right\} .
$$

Note, it should be understood throughout that we extend the valuation $\operatorname{ord}_{\ell}$ to $\mathbb{Q}(F)$ if necessary. If we let $G$ be another form of the same degree as $F$, then we say that $F \equiv_{f c} G\left(\bmod \ell^{r}\right)$ if $\operatorname{ord}_{\ell}(F-G) \geq r$.

For $n=1$ our two notions of congruence agree since (up to normalization) the $p^{\text {th }}$ Fourier coefficient is precisely the eigenvalue of $T(p)$. For $n>1$, we have the following lemma, which says that the congruence of Fourier coefficients is stronger than the congruence of eigenvalues.

Lemma 5. Let $\Sigma$ be a finite set of primes containing $\ell$ and suppose that $F$ and $G$ are eigenforms. If $F \equiv_{f c} G(\bmod \ell)$, then $F \equiv_{\Sigma} G(\bmod \ell)$.

Proof. Note, this proof follows exactly the same as the proof of Theorem A.1 in [18]. We only include it here to emphasize that we are interested in the case of arbitrary level, not just the level one case as in [18].

Define $K$ to be the compositum of $\mathbb{Q}(F)$ and $\mathbb{Q}(G)$. Let $c \in K$ so that at least one of the Fourier coefficients of $c F$ is an $\ell$-unit. Without loss of generality we replace $F$ and $G$ by $c F$ and $c G$, respectively. Let $T$ be the index of the $\ell$-unit Fourier coefficient of $F$. 
Let $t \in \mathcal{H}_{\mathbb{Z}}^{\Sigma}$. Then there exist algebraic numbers $\lambda_{F}(t), \lambda_{G}(t)$, such that $F \mid t=\lambda_{F}(t) F$ and $G \mid t=\lambda_{G}(t) G$. Define a form $H:=F-G$. Then,

$$
\lambda_{F}(t) F-\lambda_{G}(t) G=(F-G)|t=H| t .
$$

Note, by Theorem 6.1 of $[12]$ we have that $\mathbb{Q}(H \mid t) \subseteq K$. Hence,

$$
\lambda_{F}(t) a_{F}(T) \equiv \lambda_{G}(t) a_{G}(T) \quad(\bmod \nu),
$$

where $\nu$ is a prime lying above $\ell$ in $K$. Since $a_{F}(T) \equiv a_{G}(T)(\bmod \nu)$ and $a_{F}(T)$ is an $\ell$-unit, we have that $\lambda_{F}(t) \equiv \lambda_{G}(t)(\bmod \nu)$. This completes the proof.

\section{The trace operator}

In this section, we introduce the trace operator from [4] in the degree 2 setting.

Let $F \in M_{k}^{2}(N \ell, \chi)$. We define the trace of $F$ to be

$$
\operatorname{Tr}_{\Gamma_{1}^{2}(N)}^{\Gamma_{1}^{2}(N \ell)}(F):=\frac{1}{\left[\Gamma_{1}^{2}(N): \Gamma_{1}^{2}(N \ell)\right]} \sum_{\gamma \in \Gamma_{1}^{2}(N \ell) \backslash \Gamma_{1}^{2}(N)} \chi^{-1}(\gamma) F \mid \gamma
$$

where the summation is taken over a complete set of coset representatives. Note, when the levels are clear from context we will simply write $\operatorname{Tr}(F)$. The following proposition gives us a necessary result on the level of $\operatorname{Tr}(F)$.

Proposition 6. [4, Prop. 2.1] Let $F$ be as above. Then,

(1) If the conductor of $\chi$ does not divide $N$ we have

$$
\operatorname{Tr}_{\Gamma_{1}^{2}(N)}^{\Gamma_{1}^{2}(N \ell)}(F)=0
$$

(2) If the conductor of $\chi$ divides $N$ we have

$$
\operatorname{Tr}_{\Gamma_{1}^{2}(N)}^{\Gamma_{1}^{2}(N \ell)}(F) \in M_{k}(N, \chi) \text { and } \operatorname{Tr}_{\Gamma_{0}^{2}(N)}^{\Gamma_{2}^{2}(N)}(F)=\operatorname{Tr}_{\Gamma_{1}^{2}(N)}^{\Gamma_{1}^{2}(N \ell)}(F) .
$$

For our main result we will need an explicit set of representatives for $\Gamma_{1}^{2}(N \ell) \backslash \Gamma_{0}^{2}(N)$. We recall the following construction given in the proof of Theorem 4.6 in [4].

Let

$$
P:=\left\{\left(\begin{array}{cc}
A & B \\
0 & D
\end{array}\right) \in \operatorname{Sp}_{4}\left(\mathbb{F}_{\ell}\right)\right\}
$$

be the Siegel parabolic and define

$$
\omega_{j}:=\left(\begin{array}{cccc}
1_{2-j} & 0 & 0_{2-j} & 0 \\
0 & 0_{j} & 0 & -1_{j} \\
0_{2-j} & 0 & 1_{2-j} & 0 \\
0 & 1_{j} & 0 & 0_{j}
\end{array}\right) \in \operatorname{Sp}_{4}\left(\mathbb{F}_{\ell}\right)
$$

for $0 \leq j \leq 2$. Using these matrices we have the Bruhat decomposition

$$
\mathrm{Sp}_{4}\left(\mathbb{F}_{\ell}\right)=\bigsqcup_{j=0}^{2} P \omega_{j} P
$$

Furthermore, we have the Levi decomposition $P=M N$, where the Levi factor is given by

$$
\left.M:=\left\{m(A):=\left(\begin{array}{cc}
A & 0 \\
0 & T
\end{array}\right): A \in A^{-1}\right) \mathrm{GL}_{2}\left(\mathbb{F}_{\ell}\right)\right\}
$$


and the unipotent radical is given by

$$
N:=\left\{n(B):=\left(\begin{array}{cc}
1 & B \\
0 & 1
\end{array}\right):{ }^{T} B=B, B \in \operatorname{Mat}_{2}\left(\mathbb{F}_{\ell}\right)\right\} .
$$

Combining these we have that a complete set of representatives of $P \backslash P \omega_{j} P$ is given by

$$
\left\{\omega_{j} n\left(B_{j}\right) m(A):{ }^{T} B_{j}=B_{j}, B_{j} \in \operatorname{Mat}_{j}\left(\mathbb{F}_{\ell}\right), A \in P_{2, j}\left(\mathbb{F}_{\ell}\right) \backslash \mathrm{GL}_{2}\left(\mathbb{F}_{\ell}\right)\right\},
$$

where Mat $_{j}$ is embedded into Mat 2 by $B_{j} \mapsto\left(\begin{array}{cc}0 & 0 \\ 0 & B_{j}\end{array}\right)$, and

$$
P_{2, j}\left(\mathbb{F}_{\ell}\right):=\left\{\gamma \in \mathrm{GL}_{2}\left(\mathbb{F}_{\ell}\right): \gamma=\left(\begin{array}{cc}
* & * \\
0_{j, 2-j} & *
\end{array}\right)\right\} .
$$

Note, $P_{2, j}\left(\mathbb{F}_{\ell}\right)=\mathrm{GL}_{2}\left(\mathbb{F}_{\ell}\right)$ when $j \neq 1$. We can lift these representatives to representatives of $\Gamma_{0}^{2}(N \ell) \backslash \Gamma_{0}^{2}(N)$ using strong approximation, where we identify the lifts with their image modulo $\ell$. Thus, for $\omega_{j}$ satisfying

$$
\omega_{j} \equiv 1_{4} \quad(\bmod N) \text { and } \omega_{j} \equiv\left(\begin{array}{cccc}
1_{2-j} & 0 & 0_{2-j} & 0 \\
0 & 0_{j} & 0 & -1_{j} \\
0_{2-j} & 0 & 1_{2-j} & 0 \\
0 & 1_{j} & 0 & 0_{j}
\end{array}\right) \quad(\bmod \ell)
$$

we have that

$$
\left\{\omega_{j} n\left(B_{j}\right) m(A): 0 \leq j \leq 2\right\}
$$

is a complete set of representatives for $\Gamma_{0}^{2}(N \ell) \backslash \Gamma_{0}^{2}(N)$. Furthermore, we may assume for our lifted $m(A)$ that $\operatorname{det} A=1$. This gives us that $\chi^{-1}$ is trivial on our set of representatives. Using these representatives we rewrite

$$
\operatorname{Tr}(F):=F+\sum_{b=1}^{\ell-1} \sum_{A} F\left|\omega_{1} n(b) m(A)+\sum_{\substack{T \\ B=B \\(\bmod \ell)}} F\right| \omega_{2} n(B) .
$$

To complete this section, we give a more explicit expression for the last term in the trace, which we will need later. Note, since $F$ is a cusp form, we have that $F \mid \omega_{2}$ is also a cusp form. In particular, we know that the Fourier expansion is of the following form:

$$
F \mid \omega_{2}(Z)=\sum_{T \in \frac{1}{\ell} \Lambda_{2}} a(T) q^{\operatorname{tr}(T Z)}
$$

From [5] we have that

$$
\sum_{\substack{T \\ B=B \\(\bmod \ell)}} F \mid \omega_{2} n(B)=\ell^{3} \sum_{T \in \Lambda_{2}} a(T) q^{\operatorname{tr}(T Z)} .
$$

Using this we obtain

$$
\sum_{\substack{T \\
B=B \\
(\bmod \ell)}} F\left|\omega_{2} n(B)=\ell^{3} F\right| \omega_{2}\left(\begin{array}{cc}
\ell_{2} & 0 \\
0 & 1
\end{array}\right) U(\ell)
$$


We will need the following lemma, which is from [5], in the next section. We prove it here for completeness.

Lemma 7. Let $F \in S_{k}^{2}(N \ell, \chi)$ be an eigenform with associated character $\chi$ defined modulo $N$. Then, for some integer $k^{\prime}$ there exists $G \in S_{k^{\prime}}^{2}(N, \chi)$ such that $F \equiv_{f c} G$ $(\bmod \nu)$, where $\nu$ is a prime lying above $\ell$.

Proof. Note, as we are only proving this for degree 2 Siegel modular forms, we will drop the 2 from the superscript for the remainder of this proof.

By Lemma 3 we have that the Hecke operator $U(\ell)$ is an injective map from $S_{k}(N \ell)$ into itself. Thus, we can find a $g \in S_{k}(N \ell)$ such that $g \mid U(\ell)=F$. Using this $g$, define the following form:

$$
G=g\left|\left(\begin{array}{cc}
\frac{1}{\ell} \cdot 1_{2} & 0 \\
0 & 1_{2}
\end{array}\right)\right| \omega_{2}^{-1}
$$

Let $\mathcal{K}_{\ell-1}:=\ell^{2} \Theta_{L}^{2}$, where $\Theta_{L}^{2}$ is the theta series associated to an $\ell$-special lattice $L$ of rank $2 \ell-2$ and determinant $\ell^{2}$, see [6]. Then, $\mathcal{K}_{\ell-1}$ satisfies

$$
\begin{gathered}
\mathcal{K}_{\ell-1} \equiv_{f c} \mathcal{K}_{\ell-1} \mid \omega_{1} \equiv_{f_{c}} 0 \quad(\bmod \ell), \\
\mathcal{K}_{\ell-1} \mid \omega_{2} \equiv_{f c} 1 \quad(\bmod \ell) .
\end{gathered}
$$

Furthermore, $\mathcal{K}_{\ell-1}$ has integral Fourier coefficients. Applying our formula for the trace we may write

$$
\begin{aligned}
\operatorname{Tr}\left(G \mathcal{K}_{\ell-1}^{\ell^{m}}\right)= & G \mathcal{K}_{\ell-1}^{\ell^{m}}+\sum_{b=1}^{\ell-1} \sum_{A}\left(G \mathcal{K}_{\ell-1}^{\ell^{m}}\right) \mid \omega_{1} n(b) m(A) \\
& +\sum_{B} \sum_{\substack{B=B \\
(\bmod \ell)}}\left(G \mathcal{K}_{\ell-1}^{\ell^{m}}\right) \mid \omega_{2} n(B),
\end{aligned}
$$

for an arbitrary constant $m$. Our goal is to show that

$$
\operatorname{ord}_{\ell}\left(\operatorname{Tr}\left(G \mathcal{K}_{\ell-1}^{\ell^{m}}\right)\right) \rightarrow \infty \text { as } m \rightarrow \infty \text {. }
$$

To this end we will examine each piece of the summation separately. Note, throughout we will use the fact that $\operatorname{ord}_{\ell}\left(G \mid \omega_{i}\right)>-\infty$ for $i=0,1,2$, which follows from Lemma 2.1 in [23]. First,

$$
\operatorname{ord}_{\ell}\left(G \mathcal{K}_{\ell-1}^{\ell^{m}}\right)=\operatorname{ord}_{\ell}(G)+\ell^{m} \operatorname{ord}_{\ell}\left(\mathcal{K}_{\ell-1}\right),
$$

which becomes arbitrarily large as $m$ grows.

Second,

$$
\operatorname{ord}_{\ell}\left(\sum_{b=1}^{\ell-1} \sum_{A}\left(G \mathcal{K}_{\ell-1}^{\ell^{m}}\right) \mid \omega_{1} n(b) m(A)\right) \geq 1+\operatorname{ord}_{\ell}\left(G \mid \omega_{1}\right)+\ell^{m} \operatorname{ord}_{\ell}\left(\mathcal{K}_{\ell-1} \mid \omega_{1}\right),
$$

which also becomes arbitrarily large as $m$ grows. 
Third, to examine the last term of the summation we rewrite $\mathcal{K}_{\ell-1}^{\ell^{m}}=1+\ell^{m+1} X$, where $X$ is a Fourier series with integral Fourier coefficients. Then,

$$
\begin{aligned}
\sum_{\substack{T \\
B=B \\
(\bmod \ell)}}\left(G \mathcal{K}_{\ell-1}^{\ell^{m}}\right) \mid \omega_{2} n(B)= & G\left|\omega_{2}\right|\left(\begin{array}{cc}
\ell \cdot 1_{2} & 0 \\
0 & 1_{2}
\end{array}\right) U(\ell) \\
& +\ell^{m+1}\left(G \mid \omega_{2} \cdot X\right) \mid\left(\begin{array}{cc}
\ell \cdot 1_{2} & 0 \\
0 & 1_{2}
\end{array}\right) U(\ell) \\
= & F+\ell^{m+1}\left(G \mid \omega_{2} \cdot X\right) \mid\left(\begin{array}{cc}
\ell \cdot 1_{2} & 0 \\
0 & 1
\end{array}\right) U(\ell) .
\end{aligned}
$$

Note, $\operatorname{ord}_{\ell}(X) \geq 0$. Combining we have,

$$
\operatorname{ord}_{\ell}\left(F-\operatorname{Tr}\left(G \mathcal{K}_{\ell-1}^{\ell^{m}}\right) \geq m+1+\operatorname{ord}_{\ell}\left(G \mid \omega_{2}\right),\right.
$$

which goes to infinity as $m$ does. Thus, for large enough $m$ we have that

$$
\operatorname{Tr}\left(G \mathcal{K}_{\ell-1}^{\ell^{m}}\right) \equiv_{f c} F \quad(\bmod \nu),
$$

where $\nu$ is a prime lying over $\ell$ in $\mathbb{Q}(F)$.

\section{Level stripping}

In this section, we will prove the following theorem, which is our main result.

Theorem 8. Let $F \in S_{k}\left(\Gamma_{1}^{2}\left(N \ell^{r}\right)\right)$ be an eigenform with associated character $\chi$ defined modulo $\mathrm{N} \ell$ and let $\Sigma$ be the set of rational primes which divide $N \ell$. Then, for some integer $k^{\prime}>2$ there exists an eigenform $G \in S_{k^{\prime}}\left(\Gamma_{1}^{2}(N)\right)$ such that $F \equiv_{\Sigma} G$ $(\bmod \ell)$.

Proof. Note that throughout we are working with degree 2 Siegel modular forms, so we will drop the superscript. Furthermore, throughout the proof we will not be explicit about the weights of the intermediate forms, but we will make a note about the final weight $k^{\prime}$ at the end. Finally, we will take finite extensions of $\mathbb{Q}$ as needed and let $\nu$ always denote a prime lying above $\ell$.

As $\chi$ is a character modulo $N \ell$ we obtain a factorization $\chi=\omega^{i} \kappa$, where $\omega$ is the unique character of conductor $\ell$ and order $\ell-1$ and $\kappa$ is a character modulo $N$.

Let $E \in M_{k_{1}}\left(\ell, \omega^{-i}\right)$ be a form from the sequence in Theorem 1.2 of [15] such that $E \equiv_{f c} 1(\bmod \ell)$, where our congruence is in the number field containing the Fourier coefficients of $E$.

Consider the product of Siegel modular forms FE.

We first want to show that this product transforms under the action of $\Gamma_{0}\left(\ell^{r}\right) \cap$ $\Gamma_{1}(N)$. Let $\gamma \in \Gamma_{0}\left(\ell^{r}\right) \cap \Gamma_{1}(N)$. Then,

$$
\begin{aligned}
(F(Z) E(Z)) \mid \gamma & =\kappa \omega^{i}(\gamma) \omega^{-i}(\gamma) \operatorname{det}(C Z+D)^{-k-k_{1}} F(\gamma Z) E(\gamma Z) \\
& =F(Z) E(Z) .
\end{aligned}
$$

Thus, the product is a form of the desired level, character $\kappa$, and weight $k+k_{1}$. Furthermore, as $E \equiv_{f c} 1(\bmod \ell)$ we have that

$$
F E \equiv{ }_{f c} F \quad(\bmod \nu)
$$


where this congruence in the number field which contains the Fourier coefficients of both $F$ and $E$. Thus, $F E$ is an eigenform modulo $\nu$, and Lemma 5 gives us

$$
F E \equiv_{\Sigma} F \quad(\bmod \ell) \text {. }
$$

Let $\mathcal{O}_{\nu}$ be the extension of $\mathbb{Z}_{\ell}$ which has $\nu$ as its maximal ideal. As $S_{k+k_{1}}\left(\Gamma_{0}\left(\ell^{r}\right) \cap\right.$ $\left.\Gamma_{1}(N)\right)$ is a finite, free $\mathcal{O}_{\nu}$ module, we can apply the Deligne-Serre lifting lemma (Lemme 6.11, [9]) to obtain an eigenform $F_{1} \in S_{k+k_{1}}\left(\Gamma_{0}\left(\ell^{r}\right) \cap \Gamma_{1}(N)\right)$ and with character $\kappa$ such that

$$
F_{1} \equiv_{\Sigma} F \quad(\bmod \ell),
$$

where we may need to take a further field extension to obtain this congruence.

Now, consider the form $F_{1} \mid U(\ell)$. By Lemma 4 we have that

$$
F_{1} \mid U(\ell) \in S_{k+k_{1}}\left(\Gamma_{0}\left(\ell^{r-1}\right) \cap \Gamma_{1}(N)\right) .
$$

Using the commutativity of Hecke operators we see that

$$
F \mid U(\ell) \equiv_{\Sigma} F_{1} \quad(\bmod \ell),
$$

in fact, we have equality of eigenvalues away from $\ell N$. Thus, by applying the $U(\ell)$ operator $r-1$ times to $F_{1}$ we have an eigenform $F_{2} \in S_{k+k_{1}}\left(\Gamma_{0}(\ell) \cap \Gamma_{1}(N)\right)$ with character $\kappa$ such that

$$
F_{2} \equiv_{\Sigma} F_{1} \quad(\bmod \ell) .
$$

Applying Lemma 7 to $F_{2}$ we obtain a form $G \in S_{k^{\prime}}(N, \chi)$ satisfying

$$
G \equiv_{f c} F \quad(\bmod \nu) .
$$

Just as before, we use Lemma 5 and the Deligne-Serre lifting lemma a second time to obtain an eigenform $G_{1} \in S_{k^{\prime}}(N, \chi)$ such that

$$
G_{1} \equiv_{\Sigma} F \quad(\bmod \ell) .
$$

Finally, with regards to weight $k^{\prime}$, we simply note that

$$
k^{\prime} \equiv k+i \quad(\bmod \ell-1)
$$

where $\omega^{i}$ was the character obtained from the factorization of $\chi$.

\section{Application to Galois representations}

In this section, we present an application of Theorem 8 which provides evidence for a conjecture of Herzig and Tilouine.

We begin with the following result which gives the existence of a Galois representation attached to a cuspidal Siegel eigenform of degree 2 as well as the characteristic polynomial of the images of the Frobenius elements with respect to this representation. Note, this result is stated in [22], however the proof is essentially due to Laumon in [17] and Weissauer in [25, 26]. Where the last reference is necessary to conclude that the associated Galois representation is symplectic.

Theorem 9. Let $F \in S_{k}^{2}(M, \chi)$ be an eigenform. Let $K=\mathbb{Q}\left(\lambda_{F}\right)$ and let $\nu$ be a prime lying above $\ell$ in $K$. Then, there exists a continuous, semi-simple Galois representation

$$
\rho_{F, \ell}: G_{\mathbb{Q}} \rightarrow \operatorname{GSp}_{4}\left(\mathcal{O}_{K_{\nu}}\right)
$$


such that for all primes $p \nmid \ell M$ we have the characteristic polynomial of $\rho_{F, \ell}\left(\operatorname{Frob}_{p}\right)$ is given by

$$
x^{4}-\lambda_{F}(p) x^{3}+\left(p \lambda_{F}\left(p^{2}, 1\right)+\left(p^{k}+p^{k-2}\right) \chi(p)\right) x^{2}-p^{k} \lambda_{F}(p) x+p^{2 k} \chi(p),
$$

and $\rho_{F, \ell}$ is unramified at $p$.

As we have chosen a lattice so that our representation takes values in $\operatorname{GSp}_{4}\left(\mathcal{O}_{K_{\nu}}\right)$, we may form the residual representation of $\rho_{F, \ell}$ at $\ell$, i.e., the representation

$$
\bar{\rho}_{F, \ell}: G_{\mathbb{Q}} \rightarrow \operatorname{GSp}_{4}\left(\overline{\mathbb{F}}_{\ell}\right)
$$

by reducing the image of $\rho_{F, \ell}$ modulo $\nu$. If $\bar{\rho}$ is a representation which is isomorphic to $\bar{\rho}_{F, \ell}$ then we say that $\bar{\rho}$ is modular of level $M$. Note, if we need to specify that $\bar{\rho}$ arises from a form of character $\chi$, then we will simply say that $\bar{\rho}$ has character $\chi$.

With this in mind, we can ask when is a representation

$$
\bar{\rho}: G_{\mathbb{Q}} \rightarrow \operatorname{GSp}_{4}\left(\overline{\mathbb{F}}_{\ell}\right)
$$

modular?

In a partial answer to this question, Herzig and Tilouine have given sufficient conditions under which $\bar{\rho}$ is conjectured be modular. The reason this is a partial answer is that Herzig and Tilouine restrict to the ordinary setting. In order to state precisely the conjecture of Herzig and Tilouine we will need a bit of background. For more details the reader is referred to [13].

First, we say that $\bar{\rho}$ is odd if $\mu \circ \bar{\rho}(c)=-1$, where $c \in G_{\mathbb{Q}}$ is complex conjugation and $\mu$ is the similitude factor. Note, to see that this is necessary for a representation to be modular, the reader is referred to Section 9 of [24].

Second, we will need the following definition.

Definition 10. Let $F$ be a degree 2, level $M$ eigenform of weight $k$. We say that $F$ is ordinary at $\ell$ if the roots of the characteristic polynomial of $\rho_{F, \ell}$ (Frob $\ell$ ), denoted $r_{1}, r_{2}, r_{3}, r_{4}$ satisfy

$$
\operatorname{ord}_{\ell}\left(r_{1}\right)=0, \operatorname{ord}_{\ell}\left(r_{2}\right)=k-2, \operatorname{ord}_{\ell}\left(r_{3}\right)=k-1, \operatorname{ord}_{\ell}\left(r_{4}\right)=2 k-3 .
$$

Let $D_{\ell}$ be the decomposition group of $\ell$ in $G_{\mathbb{Q}}$. Let $\chi_{\ell}$ denote the $\ell$-adic cyclotomic character and for an $\ell$-adic number $u$, we set $\epsilon(u)$ to be the unramified character of $D_{\ell}$ which sends Frob $_{\ell}$ to $u$. Then, for $F$ ordinary at $\ell$ as in the definition, we have

$$
\left.\rho_{F, \ell}\right|_{D_{\ell}} \sim\left(\begin{array}{cccc}
\chi_{\ell}^{2 k-3} \epsilon\left(r_{4} / \ell^{2 k-3}\right) & * & * & * \\
0 & \chi_{\ell}^{k-1} \epsilon\left(r_{3} / \ell^{k-1}\right) & * & * \\
0 & 0 & \chi_{\ell}^{k-2} \epsilon\left(r_{2} / \ell^{k-2}\right) & * \\
0 & 0 & 0 & \epsilon\left(r_{1}\right)
\end{array}\right) .
$$

With this definition in mind, for a representation

$$
\bar{\rho}: G_{\mathbb{Q}} \rightarrow \operatorname{GSp}_{4}\left(\overline{\mathbb{F}}_{\ell}\right),
$$

we will say $\bar{\rho}$ is ordinary at $\ell$ if up to conjugation we have

$$
\left.\bar{\rho}\right|_{D_{\ell}} \sim\left(\begin{array}{cccc}
\bar{\chi}_{\ell}^{e_{3}} \epsilon\left(u_{3}\right) & * & * & * \\
0 & \bar{\chi}_{\ell}^{e_{2}} \epsilon\left(u_{2}\right) & * & * \\
0 & 0 & \bar{\chi}_{\ell}^{e_{1}} \epsilon\left(u_{1}\right) & * \\
0 & 0 & 0 & \bar{\chi}_{\ell}^{e_{0}} \epsilon\left(u_{0}\right)
\end{array}\right)
$$


where $\bar{\chi}_{\ell}$ is the reduction of $\chi_{\ell}$ modulo $\ell$, the exponents satisfy $e_{3} \geq e_{2} \geq e_{1} \geq e_{0}, \epsilon$ is as above, and $u_{3}, u_{2}, u_{1}, u_{0} \in \overline{\mathbb{F}}_{\ell}^{\times}$. We will denote such a representation as $\left(\bar{\rho},\left\{e_{j}\right\}\right)$. Note, after twisting by an appropriate power of $\bar{\chi}_{\ell}$ we may assume $e_{0}=0$ and that $e_{j} \leq j(\ell-2)$ for $j=1,2,3$. This brings us to the next definition.

Definition 11. For a representation $\left(\bar{\rho},\left\{e_{j}\right\}\right)$ as above, we say that the exponents $\left\{e_{j}\right\}$ are $\ell$-small if we can twist $\bar{\rho}$ by a power of $\bar{\chi}_{\ell}$ so that $0=e_{0} \leq e_{1} \leq e_{2} \leq e_{3}<$ $p-1$.

Furthermore, if we can write $e_{1}=k-2$ and $e_{2}=k-1$ for some integer $k \geq 2$ then we call $k$ the modular weight of $\left(\bar{\rho},\left\{e_{j}\right\}\right)$.

We are now prepared to state the following conjecture.

Conjecture 12. [13, Conj. 0] Let $\left(\bar{\rho},\left\{e_{j}\right\}\right)$ be an irreducible, odd Galois representation which is ordinary at $\ell$ and has modular weight $k$. Suppose further that the exponents $\left\{e_{j}\right\}$ are $\ell$-small. Then, $\bar{\rho}$ is modular of level $N$ with $\ell \nmid N$.

Note, we have specialized to our setting, as the conjecture is actually stated in the more general setting of vector-valued modular forms.

As evidence for this conjecture, we can state the following corollary, which follows from Theorem 8 .

Corollary 13. Suppose that $\bar{\rho}$ is modular of level $\ell^{r} N$ and character $\chi$ of conductor $\ell N$ with $\ell \nmid N$. Then, $\bar{\rho}$ is modular of level $N$.

Proof. Suppose that $\bar{\rho}$ arises from $F \in S_{k}\left(\ell^{r} N\right)$. Then, we can apply Theorem 8 to obtain a representation $\bar{\rho}^{\prime}$ of level $N$ such that the characteristic polynomials of $\bar{\rho}\left(\right.$ Frob $\left._{p}\right)$ and $\bar{\rho}^{\prime}\left(\right.$ Frob $\left._{p}\right)$ are equal for all $p \nmid \ell N$. Thus, the characteristic polynomials of $\bar{\rho}$ and $\bar{\rho}^{\prime}$ are equal everywhere by the Chebotarev Density Theorem. Thus, the Brauer-Nesbitt Theorem gives us that $\bar{\rho}$ is isomorphic to $\bar{\rho}^{\prime}$.

\section{Future work}

In this section, we mention some open problems related to generalizing our result.

(1) Let $F$ be the eigenform from Theorem 8 with corresponding character of conductor $N \ell^{r}$ for $r \geq 1$. Is there a way to construct an eigenform with corresponding character of conductor $N \ell$ whose eigenvalues are congruent to those of $F$ away from the level?

In the proof of Theorem 1, Ribet is able to twist the form so that the conductor of the character is lowered. However, this is for elliptic modular forms, and the generalization of this twisting to the degree 2 setting is not obvious. If one could answer this question affirmatively then we could relax our restriction on the character of $F$.

(2) Can we find an Eisenstein series of arbitrary degree analogous to the one used in the proof of Theorem 8, i.e., can we find an Eisenstein series, $E$, of degree $n$, level $\ell$, and character $\omega^{-i}$ (as in the proof) such that $E \equiv_{f c}$ $1(\bmod \ell)$ ? 
Note, this is closely related to Problem 4.1 from [15]. If one could answer this question affirmatively then we could generalize Theorem 8 to arbitrary degree.

\section{Acknowledgments}

The author would like to thank Jim Brown for suggesting this problem and for the many helpful conversations. Also, the author would like to thank Siegfried Böcherer and Shoyu Nagaoka for providing a preprint, which was crucial to this work.

\section{References}

[1] A. Andrianov, On diagonalization of singular Frobenius operators on Siegel modular forms, Amer. J. Math. 125 (2003), 139-165.

[2] —, Introduction to Siegel modular forms and Dirichlet series, Universitext, Springer, New York, NY (2009).

[3] S. Böcherer, On the Hecke Operator $U(p)$, J. Math. Kyoto Univ. 45(4) (2005), $807-829$.

[4] S. Böcherer, J. Funke and R. Schulze-Pillot, Trace operator and theta series, J. Number Theory 78 (1999), 119-139.

[5] S. Böcherer and S. Nagaoka, On p-adic properties of Siegel modular forms, preprint.

[6] - On Siegel modular forms of level $p$ and their properties mod $p$, Manuscripta Math. 132 (2010), 501-515.

[7] R. Coleman and J.-F. Voloch, Companion forms and Kodaira-Spencer theory, Invent. Math. 110 (1992), 263-282.

[8] P. Deligne, Formes modulaires et représentations $\ell$-adiques, Ann. Scient. Ec. Norm. Sup. 7 (1974), 507-530.

[9] P. Deligne and J.-P. Serre, Formes modulaires de poids 1, Ann. Sci. Ec. Norm. Sup. 7 (1974), $507-530$.

[10] F. Diamond, The refined conjecture of Serre, in (J. Coates and S.-T. Yau, eds.), 'Elliptic Curves and Fermat's Last Theorem', 172-186, International Press (1997).

[11] B. Gross, A tameness criterion for Galois representations associated to modular forms $(\bmod p)$, Duke Math. J. 61 (1990), 445-516.

[12] J. Hafner and L. Walling, Explicit action of Hecke operators on Siegel modular forms, J. Number Theory 93 (2002), 34-57.

[13] F. Herzig and J. Tilouine, Conjecture de type de Serre et formes compagnons pour GSp 4 , To Appear J. Reine Angew. Math 676 (2013), 1-32.

[14] C. Khare and J.-P. Wintenberger, Serres modularity conjecture (I), Invent. Math. 178 (2009), 485-504.

[15] T. Kikuta, On p-adic Siegel modular forms of non-real Nebentypus of degree 2, Acta Arith. 152 (2012), 175-183.

[16] N. Kurokawa, On Siegel eigenforms, Proc. Japan Acad. Ser. A 57 (1981), 47-50.

[17] G. Laumon, Fonctions zéta des variétés de Siegel de dimension trois, Astérisque 302 (2005), $1-66$.

[18] S. Mizumoto, Congruences for Fourier Coefficients of Lifted Siegel Modular Forms I: Eisenstein Lifts, Abh. Math. Sem. Univ. Hamburg 75 (2005), 97-120.

[19] K. Ribet, On Modular Representations of $\operatorname{Gal}(\overline{\mathbb{Q}} / \mathbb{Q})$ arising from modular forms, Invent. Math. 100 (1990), 431-476.

$[20] \longrightarrow$, Report on Mod $\ell$ Representations of $G a l(\overline{\mathbb{Q}} / \mathbb{Q})$, in Motives, Vol. 55 of Proc. Symp. Pure Math, 639-676, American Mathematical Society, Providence, RI 1994.

[21] J.-P. Serre, Sur les représentations modulaires de degré 2 de $G a l(\overline{\mathbb{Q}} / \mathbb{Q})$, Duke Math. J. 54(1) (1987), 179-230.

[22] C. Skinner and E. Urban, Sur les deformations p-adiques de certain representations automorphes, J. Inst. Math. Jussieu 5 (2006), 629-698. 
[23] R. Taylor, On congruences between modular forms, Ph.D. thesis, Princeton University 1988.

[24] J. Tilouine, Deformations of Galois representations and Hecke algebras, Narosa Publishing House 1996.

[25] R. Weissauer, Four dimensional Galois representations, Astérisque 302 (2005) 67-150.

[26] - Existence of Whittaker models related to four dimensional symplectic Galois representations, in Modular Forms on Schiermonnikoog, Cambridge University Press, 2008, 285-310.

Department of Mathematical Sciences, Clemson University, Clemson, SC 29634, USA E-mail address: rkeaton@clemson.edu http://people.clemson.edu/ rkeaton 